УДК: 620.173:538.112:539.3

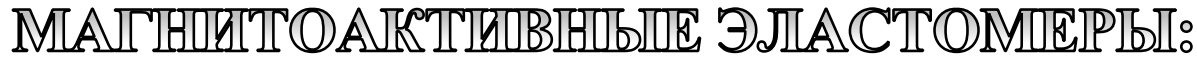

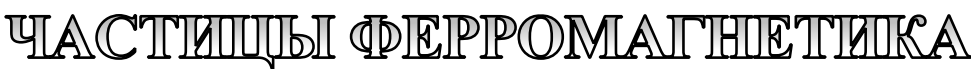

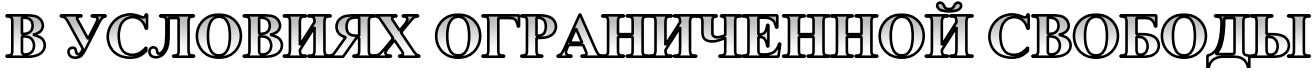

Ю.Л. Райхер, Институт механики сплошных сред УрО РАН

М.В. Ваганов, Институт механики сплошных сред УрО РАН

\section{Для цитирования:}

Райхер Ю.Л., Ваганов М.В. Магнитоактивные эластомеры: частицы ферромагнетика в условиях ограниченной свободы // Вестник Пермского федерального исследовательского центра. - 2021. - № 1. C. 6-18. https://doi.org/10.7242/2658-705X/2021.1.1

В статье приводятся некоторые результаты исследовательской работы, выполненной в 2017-2019 гг. С одной стороны, авторы не ставили целью вдаваться в детали, с другой стороны, не хотели безоглядно жертвовать строгостью в пользу доступности. По этой причине в вводной части текста (разделы 1-3) для удобства читателя представлен некоторый минимум сведений из классической физики однодоменных частиц. Отметим также, что настоящая статья - первое изложение на русском языке материала, прежде публиковавшегося только в международных журналах.

Mobilis in mobile (Подвижный в подвижном) Ж. Верн «20 000 лье под водой».

\section{1. Введение. \\ Модель Стонера-Вольфарта однодоменной частицы}

В 1948 году профессор Эдмунд Клифтон Стонер и его ассистент Эрих Петер Вольфарт - физики-теоретики, работавшие в Университете Лидса (Великобритания), опубликовали в Proceedings of the Royal Society статью под не слишком выразительным названием «A mechanism of magnetic hysteresis in heterogenous alloys» (Механизм магнитного гистерезиса в неоднородных сплавах)»[1].

Работа была посвящена объяснению свойств постоянных магнитов, которые к тому времени уже давно и широко применялись на практике. Главным качеством, интересовавшим потребителей, была, конечно, «сила» магнита, то есть его способность при минимальном размере удерживать максимальную массу магнитомягкого ферромагнетика (например, при погрузке железного лома) или же наиболее эффективно притягивать / отталкивать другой подобный магнит (например, в магнитном подвесе).

Было хорошо известно, что вопрос такой оптимизации не решается в рамках чистого материаловедения, то есть лишь подбором ингредиентов сплава. Важнейшую роль играет режим намагничивания. В самом деле, независимо от состава, выплавленный образец никакой постоянной намагниченностью не обладает и постоянным магнитом в привычном смысле никак не является. Это свойство он полу- 
чает, побывав в стороннем (внешнем) магнитном поле $H$, которое перестраивает доме́нную структуру образца.

Нужное поле создают посредством катушки с током (соленоид), причем электродинамика еще с начала XIX века благодаря трудам Андре-Мари Ампера - «Ньютона электричества», по оценке Джемса Кларка Максвелла - знает, как рассчитывать величину (напряженность) поля. На тех же раз навсегда установленных принципах работают и приборы, измеряющие намагниченность: магнитометры. По их показаниям можно сразу определить, насколько высокую намагниченность $M$ приобрел образец, то есть насколько хорошим магнитом он стал после воздействия поля.

Таким образом, с помощью относительно несложной техники можно намагнитить испытуемый образец и охарактеризовать его графиком $M(H)$. Типичные примеры можно найти во многих учебниках по физике магнитных явлений, см. рис. 1, взятый из [2]. Для всех постоянных магнитов кривая $M(H)$ имеет вид петли, где положительное и отрицательное направления оси абсцисс соответствуют магнитному полю, приложенному в разных направлениях вдоль одной и той же линии.

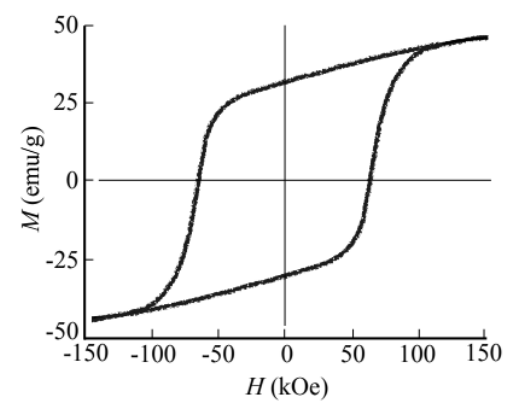

Рис. 1. Петля гистерезиса образияа Sm-Co, отожженного при 973 K, воспроизведено по [2]
Замечательным свойством этого графика является его двузначность: в некотором интервале вокруг начала координат (точка $H=0$ ) одному и тому же значению приложенного поля соответствуют два значения намагниченности: положительное и отрицательное. То есть при данной величине и направлении поля магнит может иметь различную полярность; по краям указанного интервала есть еще и участки, где полярность одинакова, но значения намагниченности сильно различаются. Только на дальних концах оси поля петля вырождается в единственную линию, которая затем идет практически горизонтально. Иными словами, в сильном поле намагниченность перестает зависеть от величины приложенного поля; эти области называются участками насыщения. Ко времени выхода работы Стонера и Вольфарта эмпирических знаний о петлях $M(H)$ различных материалов было накоплено более чем достаточно. Проблема, однако, была с ясным пониманием происхождения петель и, тем более, с их количественным описанием, а значит, с возможностью заранее рассчитать параметры петли. Между тем техника чрезвычайно нуждалась в таких расчетах, поскольку критерий качества магнита - так называемое «энергетическое произведение» - был уже давно установлен эмпирически; для этого использовались некоторые характерные значения $M$ и $H$, взятые с графика петли. Однако без теории для того, чтобы узнать величину «энергетического произведения» и другие важные свойства магнита, не было другого пути, кроме как измерять зависимость $M(H)$ - полностью или значительную его часть - в реальности для каждого конкретного случая .

\footnotetext{
* Рецензент статьи сделал(а) важное замечание относительно величин, используемых для характеристики магнитного поля, справедливо указав, что понятие напряженности этого поля отсутствует, например, в школьном курсе физики. С рациональностью современного подхода трудно спорить. Действительно, с фундаментальной точки зрения напряженность поля $\boldsymbol{H}$ - это вспомогательная величина, определяемая через реально измеримую характеристику - индукцию поля $\boldsymbol{B}$. Соотношения, связывающие $\boldsymbol{B}$ и $\boldsymbol{H}$, не сложны и их можно найти в любом учебнике по общей физике, однако их описание не входит в задачу настоящей статьи. Между тем, в литературе по макроскопическому магнетизму величина $\boldsymbol{H}$ повсеместно используется, так как дает удобную запись основных уравнений. Мы не стали переводить немногие используемые нами формулы и графики из $\boldsymbol{H}$-представления в термины $\boldsymbol{B}$ не только для сохранения этого удобства, но, главным образом, потому что целью нашей статьи является описание предмета исследования в целом. А для этого достаточно всего лишь понимать, что увеличение $H$ (то есть модуля вектора $\boldsymbol{H}$ ) означает усиление магнитного поля, в каких бы единицах оно ни измерялось.
} 
Теоретическая модель процесса намагничивания, предложенная Стонером и Вольфартом, обескураживающе проста. Базовым элементом служит одна единственная частица ферромагнетика, намагниченная однородно, то есть находящаяся в однодоменном состоянии. Такую систему характеризует единый вектор магнитного момента $\boldsymbol{\mu}$, длину которого $\mu=M V$ (где $V$ - объем частицы) приложенное поле не может изменить, так что вся доступная вектору $\boldsymbol{\mu}$ свобода движений исчерпывается поворотами, к которым его вынуждает приложенное поле.

Принципиальным свойством модельной частицы является ее магнитная анизотропия. В простом варианте (он использован в оригинальной работе) анизотропия является одноосной. Это предположение устанавливает, что в частице есть «встроенная» двусторонняя ось, вдоль которой вектор $\boldsymbol{\mu}$ смотрит в том случае, когда внешнее поле отсутствует. Двусторонность оси означает, что оба ее направления равноправны, и поэтому энергия магнитного момента в любой из двух возможных (антипараллельных друг другу) ориентаций имеет одно и то же значение. По этой причине, хотя в формальном описании удобно указывать ось анизотропии с помощью единичного вектора $\boldsymbol{n}$, все формулы, выражающие физически наблюдаемые величины, оказываются квадратичными по компонентам $\boldsymbol{n}$, то есть безразличными (инвариантными) к тому, какое из направлений оси анизотропии произвольно выбрано в качестве положительного.

В следующем разделе мы продемонстрируем основные свойства модели Стонера-Вольфарта (СВ). Здесь же отметим, что эта теоретическая модель сразу после своего появления стала безусловной «классикой» в физике магнитных явлений. Она обеспечила колоссальный скачок в физике и технике постоянных магнитов и стала фундаментом всей физики магнитной записи, о каком бы типе носителей ни шла речь. Число литературных ссылок на модель СВ исчисляется многими десятками тысяч. И хотя впоследствии
Эдмунд Стонер (его фамилия на русском иногда транскрибируется как Стоунер) написал еще и ряд замечательных работ по микроскопической теории магнетизма, атомной и астрофизике, именно эта единственная феноменологическая работа поставила его в ряд самых знаменитых физиков-теоретиков в области физики магнетизма [3, 4].

Модель СВ не выходит из употребления до настоящего времени. Обладая подкупающей простотой, она при минимуме затрат на знакомство с ней выстраивает фундаментальное понимание огромной совокупности процессов намагничивания в разнообразных магнитных материалах, число которых все расширяется.

\section{2. Петля магнитного}

\section{гистерезиса однодоменной частицы.}

\section{Аналитическое описание}

Все основные особенности поведения частицы $\mathrm{CB}$ в магнитном поле следуют из выражения для ее энергии. Эта функция, которую можно представить в виде

$$
U=-\mu H(\boldsymbol{e h})-K V(\boldsymbol{e n})^{2},
$$

содержит всего два вклада.

Первый из них - энергия взаимодействия магнитного момента с приложенным полем $\boldsymbol{H}=H \boldsymbol{h}$, где $H-$ величина поля, а $\boldsymbol{h}$ - единичный вектор его направления. Таким образом, для того, чтобы инвертировать поле $(\boldsymbol{H} \Rightarrow-\boldsymbol{H})$ в формуле (1) достаточно заменить $H$ на $-H$. Направление магнитного момента частицы $\boldsymbol{\mu}$ (напомним, что длина $\mu$ этого вектора не меняется) обозначает единичный вектор $\boldsymbol{e}$, то есть $\boldsymbol{\mu}=\mu \boldsymbol{e}$. Второе слагаемое в формуле (1) выражает взаимодействие магнитного момента частицы с еe осью анизотропии $\boldsymbol{n}$, а материальный параметр $K$ называется объемной плотностью энергии анизотропии.

Вспоминая, что скалярное произведение единичных векторов - это косинус угла между ними, легко понять, что функция $U$ зависит от двух углов, которые на рис. 2 обозначены через $\vartheta$ и $\psi$, а именно $\quad(\boldsymbol{e h})=\cos \vartheta, \quad(\boldsymbol{e n})=\cos \psi ;$ 


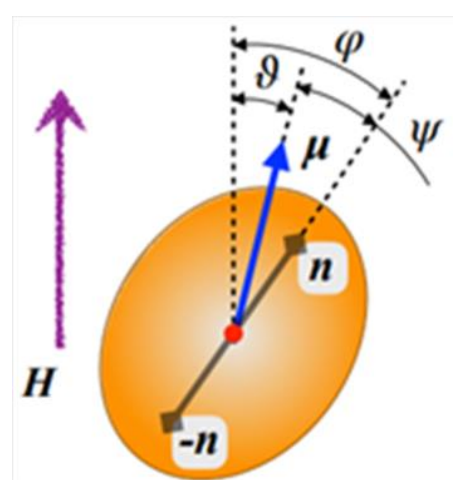

Рис. 2. Выбор обозначений для углов

как указано выше, вектор $\boldsymbol{e}$ - это вектор направления магнитного момента $\boldsymbol{\mu}$, показанного на рис. 2.

Рассматривая формулу (1) в отсутствие внешнего поля $(H=0)$, видим, что в этой ситуации минимум магнитной энергии достигается при ориентации вектора магнитного момента вдоль любого из направлений оси анизотропии (ее также называют осью легкого намагничивания частицы): $\boldsymbol{e} \| \boldsymbol{n}$ или $\boldsymbol{e} \| \boldsymbol{- n}$. В присутствии поля, для того чтобы определить равновесное направление вектора $\boldsymbol{e}$ (то есть $\boldsymbol{\mu})$, нужно найти минимум функции

$$
U(\vartheta, \psi) / K V=-2 \xi \cos \vartheta-\cos ^{2} \psi,
$$

где параметр $\xi=\mu H / 2 K V$ можно рассматривать как безразмерную величину приложенного поля или представлять его в виде отношения двух магнитных полей: внешнего и так называемого поля анизотропии $H_{A}=2 K V / \mu$, так что $\xi=H / H_{A}$.

Очевидно, что в слабом поле $\left(H \ll H_{A}\right)$ или $\xi \ll 1$ вектор $\boldsymbol{e}$ лишь слегка отклоняется от оси анизотропии $\boldsymbol{n}$, а в сильном $\left(H \gg H_{A}\right)$ или $\xi \gg 1$ - устанавливается практически параллельно направлению поля независимо от того, как расположена ось легкого намагничивания частицы.

В полях, где нет явного превышения одного фактора над другим, направление магнитного момента должно сильно зависеть как от параметра $\xi$, так и от начального угла $\vartheta_{0}$, который магнитный момент имел до включения поля. Примем положительное направление поля за ось системы координат и введем угол $\varphi$ для обозначения в ней направления оси анизотропии. Тогда функция (2) примет вид

$$
U(\vartheta, \varphi) / K V=-2 \xi \cos \vartheta-\cos ^{2}(\varphi, \vartheta),
$$

где все углы теперь отсчитываются от единой оси - направления поля.

Важные свойства функции (3) проще всего продемонстрировать для случая, когда магнитное поле параллельно оси анизотропии частицы, то есть $\varphi=0$. После такого упрощения из (3) имеем

$$
U(\vartheta) / K V=-2 \xi \cos \vartheta-\cos ^{2} \vartheta .
$$

Профиль функции (4) показан на рис. 3. Из него видно, что до тех пор пока параметр $\xi$ остается меньшим единицы, потенциал $U(\vartheta)$ имеет два минимума. Иными словами, когда приложенное поле относительно мало, то магнитный момент будет находиться в той ориентации (одной из двух возможных), которую он занимал до включения поля. Если начальная ориентация совпадает с направлением поля (случай $\vartheta_{0}=0$ ), то этот минимум с ростом $\xi$ только углубляется и у вектора $\boldsymbol{e}$ нет причин его покинуть. Совсем по-иному выглядит ситуация для магнитного момента, направленного антипараллельно приложенному полю $\left(\vartheta_{0}=180^{\circ}\right)$.

На рис. 3 положение такого магнитного момента изображено крупной точкой, лежащей в минимуме потенциала на правом краю графиков. По мере роста поля минимум становится более пологим, но до тех пор, пока $\xi<1$, потенциальная яма (potential well) как таковая существует и вектор $\boldsymbol{e}$ сохраняет свое положение. Однако при наступлении равенства $\xi=1$ $\left(H=H_{A}\right)$ указанный минимум исчезает. Это означает, что положение магнитного момента в ориентации $\vartheta=180^{\circ}$ стано-

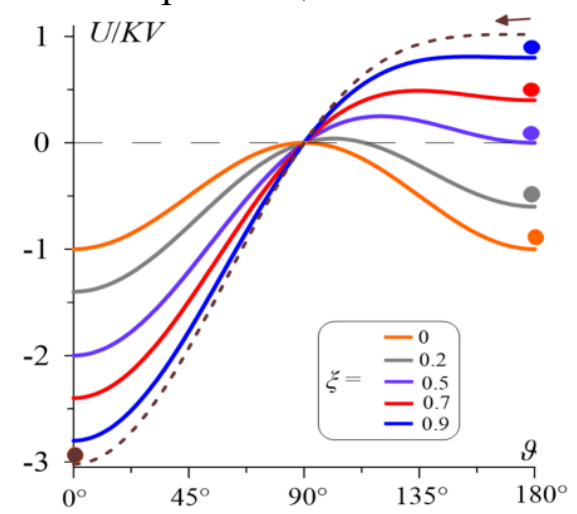

Рис. 3. Угловая зависимость потенщиала $U$ в поле, направленном вдоль оси легкого намагничивания частииь 
вится абсолютно неустойчивым и он скачком переключается в положение $\vartheta=0^{\circ}$, то есть устанавливается параллельно приложенному полю. Такой переход - он соответствует штриховой линии на рис. 3 - приводит к перебросу (переключению) угла ориентации магнитного момента, как это показано на рис. 4.

Отметим, что для рассматриваемого случая величина $\cos \vartheta$ - это проекция безразмерного магнитного момента $\boldsymbol{e}$ частицы на направление поля $\boldsymbol{h}$. Используя приведенные выше представления, легко построить график, показывающий, как изменяется эта проекция, если к частице приложить циклически изменяющееся поле большой амплитуды. Действительно, зависимость $\cos \vartheta$ от $\xi$ имеет вид прямоугольной петли, представленной на рис. 5. Стрелки на графике показывают направление изменения намагниченности при росте и убывании поля; вертикальные линии соответствуют инверсии (переключению) направления магнитного момента при $|H|=H_{A}$.

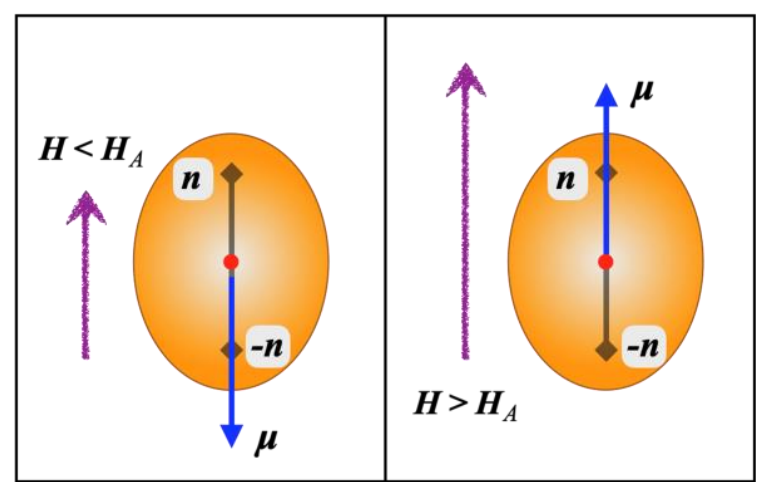

Рис. 4. Магнитное переключение частицы под действием внешнего поля, превышающего поле анизотропии. Переход от левого рисунка к правому соответствует перемещению представляющей точки на рис. 1 с правого края на левый

\section{3. Петля магнитного}

гистерезиса однодоменной частицы. Графические представления

На рис. 5 показано, что в интервале $-H_{A} \leq H \leq H_{A}$ направление магнитного момента частицы, то есть ее намагниченность, зависит от предыстории процесса: определяется не только текущей величиной приложенного поля, но и тем, в каком состоянии частица находилась на

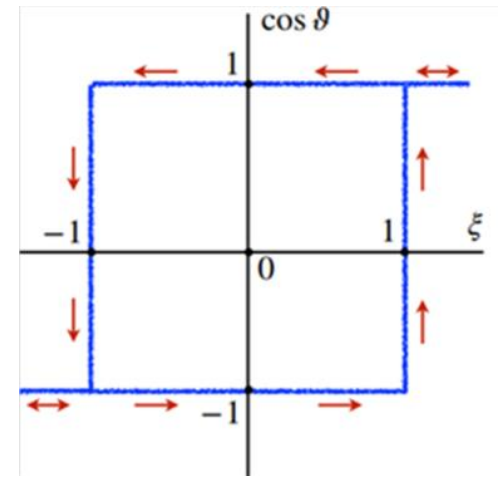

Рис. 5. Петля намагничивания частиџы СВ в безразмерных координатах

предшествующих стадиях процесса. Промежуток $-H_{A} \leq H \leq H_{A}$ на рис. 5 называется интервалом бистабильности, а изображенное поведение намагниченности гистерезисным поведением. Гистерезисные явления и петлевые диаграммы типа см. рис. 5 встречаются во многих областях физики, например, к этому типу относится фазовый переход жидкость - твердое тело, там роль управляющего параметра может играть температура, а откликом среды служить удельный объем. Однако в физику магнитных частиц предельно ясное понимание намагничивания как гистерезисного процесса привнесли именно Э. Стонер и Э. Вольфарт, поэтому термин «пе́тли Стонера-Вольфарта» уже давно превратился в стандартный.

Так произошло, прежде всего, потому, что модель СВ стала теоретическим фундаментом для всей физики магнитной записи (см., например, книгу [5]). Уже из диаграммы рис. 5 ясно видно, что частица с характеристиками $\cos \vartheta= \pm 1$ полностью соответствует требованиям к элементу, кодирующему двоичную запись. Действительно, есть два физически различных в равной степени устойчивых состояния - параллельность и антипараллельность ориентации магнитного момента заданному направлению - и известен способ переключения элемента между этими состояниями.

Взятый для иллюстрации случай $\pm \boldsymbol{e} \| \boldsymbol{h}$, то есть $\varphi=0$, конечно, не исчерпывает всех возможных вариантов взаимной ориентации оси анизотропии частицы и приложенного поля. Используя фор- 
мулу (3), расчет петель СВ можно провести для любого угла $\varphi$ - наклона оси легкого намагничивания к полю [1]. На рис. 6 показано, как трансформируются контуры кривых $\cos \vartheta(\xi)$ в зависимости от $\varphi$.

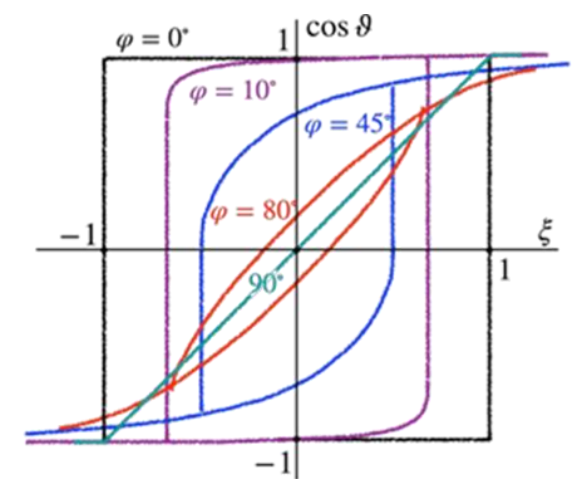

Рис. 6. Петли намагничивания частицыь СB при различных наклонах оси анизотропии

к направлению приложенного поля

Как видно, графики симметричны относительно начала координат, то есть определяющей величиной является абсолютная величина поля: $|\xi|$. В зависимости от угла $\varphi$ скачок происходит при различных значениях $|\xi|$ и имеет различную величину. Диапазон полей переключения лежит в границах $0,5 \leq \xi \leq 1$, минимальному полю отвечает $\varphi=45^{\circ}$, а максимальному - концы интервала $\left[0^{\circ}, 90^{\circ}\right]$. Однако между полуинтервалами $\left[0^{\circ}, 45^{\circ}\right]$ и $\left[45^{\circ}, 90^{\circ}\right]$ имеется существенная разница. На первом из них величина скачка намагниченности - его мерой служит длина вертикальных отрезков на рис. 6 - увеличивается с уменьшением угла $\varphi$, а в другом - уменьшается с увеличением $\varphi$, стремясь к нулю; так, например, при $\varphi=80^{\circ}$ имеющийся скачок уже близок к пределу разрешения графика.

Очевидно, что петли намагниченности однодоменной частицы, следующие из модели $\mathrm{CB}$, лишь отдаленно похожи на экспериментально наблюдаемые кривые циклического намагничивания макроскопических образцов, подобных изображенной на рис. 1. Все в той же работе [1] был сделан следующий шаг - образец ферромагнетика был представлен как система (assembly) из множества отдельных частиц $\mathrm{CB}$, направления осей легкого намагничивания кото- рых распределены по случайному закону внутри полного телесного угла. Суммарный магнитный момент такой системы в отсутствие поля фактически равен нулю вследствие усреднения. Действительно, хотя магнитный момент каждой частицы постоянен и лежит вдоль ее оси анизотропии $(\boldsymbol{e} \| \boldsymbol{n}$ или $\boldsymbol{e} \|-\boldsymbol{n})$, но оси частиц, входящих в систему, а значит и их магнитные моменты, разбросаны по всем направлениям и компенсируют друг друга.

Приложенное поле $\boldsymbol{H}$ - назовем положительным то направление, с которого начинается цикл намагничивания, - по мере увеличения все сильнее упорядочивает магнитные моменты. В том числе, при достаточной величине $\left(H>\frac{1}{2} H_{A}\right)$ оно переключает и те, что в начальном состоянии имели отрицательные проекции на поле: $(\boldsymbol{e h})<1$. В пределе поле положительного направления доводит общий магнитный момент системы до максимально возможного значения (насыщение). При снижении поля, пока оно убывает до нуля, магнитные моменты приходят к равновесным ориентациям $(\boldsymbol{e} \| \boldsymbol{n})$. Однако это состояние системы не совпадает с исходным. В самом деле, те магнитные моменты, что сначала имели отрицательную проекцию на поле, теперь занимают положение вдоль того из направлений оси легкого намагничивания, которое составляет с $\boldsymbol{h}$ угол, меньший $90^{\circ}$. Иными словами, магнитный момент каждой частицы «оседает» в том минимуме энергии, к которому он оказался ближе в угловом пространстве. Хотя угловой разброс осей легкого намагничивания не изменился, теперь каждый магнитный момент имеет неотрицательную проекцию на направление, в котором было приложено поле. В результате, после первого возрастания $H$ (напомним, что в безразмерном виде поле выражается в единицах параметра $)$ ) от нуля до некоторого $H_{\max }$ и последующего возврата $H$ к нулевому значению система оказывается намагниченной.

Цикл намагничивания системы случайно ориентированных одноосно-анизотроп- 
ных частиц, впервые рассчитанный в [1], представлен графиком рис. 7. Линия, выходящая из центра координат, соответствует «затравочному» намагничиванию, когда поле приложено впервые и в положительном направлении. Эта траектория реализуется только один раз: при снижении поля намагниченность системы следует верхней ветви графика и приходит в точку $\cos \vartheta \approx 0,5$. Иными словами, остаточная намагниченность системы равна половине максимальной, достигаемой в насыщении.

Дальнейший ход кривой рис. 7 (обозначен стрелками) вполне понятен. Поле отрицательного направления сначала «наклоняет» магнитные моменты частиц в свою сторону, а затем (при $|H|>\frac{1}{2} H_{A}$ ) начинает последовательно переключать частицы по достижении ими порога. Изменение намагниченности при возврате поля от значения $-H_{\max } \kappa$ нулю, а затем к $H_{\max }$ протекает ровно по тому же сценарию, как это было в первой половине цикла с той только разницей, что переключение частиц происходит в обратном направлении, и поэтому любая точка нижней ветви является отражением относительно начала координат соответственной точки на верхней ветви.

Петля магнитного гистерезиса рис. 7 уже очень похожа на реальную кривую рис. 1. Многочисленность частиц в системе при хаотическом разбросе их осей приводит к тому, что элементарные (одночастичные) скачки усредняются. На кривой намагничивания системы отсутствуют вертикальные участки (ср. с рис. 6), а ветви в области однозначности

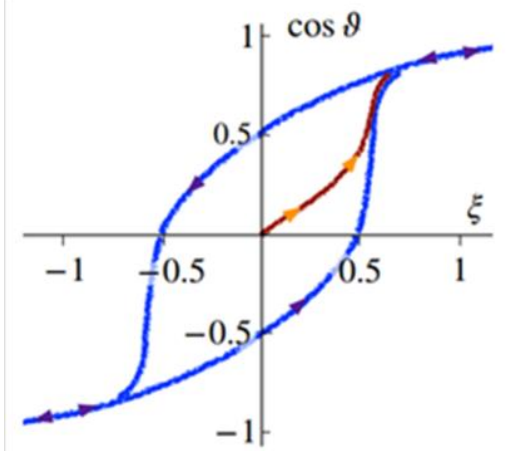

Рис. 7. Петля намагничивания системы случайно ориентированных частии $C B$ (вне петли) имеют заметный наклон, то есть предсказывают не скачкообразный, а плавный выход на насыщение, как это и бывает в действительности.

Таким образом, модель $\mathrm{CB}$, в которой магнитный отклик каждой частицы прост и понятен, оказывается способной воспроизвести кривые намагничивания больших образцов, несмотря на то, что в реальном материале имеется большое количество совершенно не учитываемых ею факторов: магнитостатическое и механическое взаимодействия между соседними элементами магнитной и кристаллической структуры, дефекты кристаллической решетки, посторонние примеси и многие другие. Естественно, что для учета этих факторов модель многократно подвергалась усовершенствованиям и до сих пор используется как база для модификаций и уточнений, специализированных для конкретных типов микро- и нанодисперсных магнитных систем. Важно, однако, что во всех этих случаях подразумевается, что частицы полностью лишены механической свободы, так как заключены в неподвижную твердую немагнитную матрицу.

\section{4. Частицы получают свободу: композиты типа МАЭ}

Краткое описание классической модели $\mathrm{CB}$, представленное в разделах 1-3-это, на самом деле, длинное, но необходимое предисловие к изложению оригинальных результатов. Без этих разъяснений нельзя понять, в чем ключевой момент новизны нашей работы. Но, прежде, еще немного необходимой информации.

Предметом нашего изучения в целом являются так называемые магнитоактивные эластомеры (МАЭ). Этот еще только входящий в употребление термин означает композиционные материалы, в которых имеются два основных компонента: мягкий полимер (матрица) и какой-либо ферромагнетик в состоянии микродисперсного порошка (наполнитель). Наполнитель смешивается с прекурсором матрицы (олигомером), после чего олигомерные молекулы сшиваются в полимерную 
сетку. Таким образом, в готовом МАЭ каждая микронная частица ферромагнетика «вклеена» в макромолекулярное окружение и достаточно прочно связана с ним.

Вот эта структура и наделяет МАЭ смарт-способностями: их механикой можно дистанционно управлять, используя внешнее магнитное поле. В самом деле, буквально все на свете материалы, кроме ферромагнитных металлов и ферритов, «прозрачны» для поля, поэтому рабочий элемент из МАЭ не нуждается в прямом контакте с управляющим магнитом или соленоидом. Манипулировать МАЭ можно через стенку / футляр / оболочку; единственным ограничением является лишь факт ослабления поля с расстоянием.

Физический принцип управления МАЭ заключается в следующем. Поле, приложенное к такому образцу, непосредственно воздействует только на частицы ферромагнитного наполнителя, магнитная чувствительность матрицы пренебрежимо мала. Появляющиеся под влиянием поля магнитостатические силы и моменты сил стремятся переместить и повернуть каждую частицу так, чтобы минимизировать магнитную потенциальную энергию всей системы. В случае твердой матрицы окружение частицы полностью компенсирует эти силовые воздействия, поэтому структура системы, сформировавшаяся по окончании ее синтеза, остается неизменной; любые смещения / повороты частиц исключены.

В МАЭ складывается иная ситуация: благодаря эластичности матрицы частицы, вынуждаемые магнитными силами, имеют возможность перемещаться внутри нее. Таким образом, если в твердых дисперсных магнетиках подвижны только магнитные моменты внутри частиц (см. выше о переключениях), то в МАЭ пространственная свобода присуща также и самим частицам как механическим объектам.

С другой стороны, легко понять, что свобода частиц в МАЭ всегда ограничена. Действительно, сдвиг и/или поворот частицы относительно ее начального (равновесного) положения возмущает эластомер- ную матрицу. Там возникают упругие напряжения, и они тем сильнее, чем значительнее деформация, созданная частицей. В конечном счете, возрастающее упругое сопротивление уравновешивает магнитные силы и движение частиц в материале останавливается. Отсюда ясно, что равновесная структура МАЭ в присутствии поля отличается от той начальной, что существовала в нем без поля: частицы находятся в иных положениях и их магнитные моменты направлены по-другому. Главным же итогом вызванной полем внутренней (мезоскопической) перестройки является то, что ее совокупный результат проявляется макроскопически. В первую очередь, это изменение формы образцов, например, изгиб стрежней, выпучивание мембран, растяжение / сжатие и другие варианты формоизменения 3D-объектов.

Важно, что все указанные изменения обратимы. При выключении поля магнитные силы/ моменты сил обращаются в нуль, так что накопленные упругие напряжения, не встречая противодействия, переводят матрицу в исходное состояние. В результате все частицы возвращаются на свои места, а образец восстанавливает свою начальную форму. При соблюдении некоторых простых условий остаточные деформации МАЭ, хотя и имеют место, но оказываются очень малыми.

Именно магнитомеханические эффекты описанного типа делают МАЭ предметом пристального интереса со стороны специалистов-прикладников. В настоящее время МАЭ исследуются / используются в качестве рабочих элементов в роботике и акторике, вибрационной и виброзащитной технике, лабораторном приборостроении различных направлений, медицинской технике.

Стремление создать МАЭ с наиболее подходящими для приложений свойствами привело к тому, что, наряду с уже ставшими классическими композитам на основе порошков карбонильного железа дисперсностью 2-5 мкм [6-9], появились материалы, где наполнителем служат частицы высококоэрци- 
тивных (магнитожестких) интерметаллидов семейства $\mathrm{NdFeB}$ (Неомакс) [2]. Они на порядок крупнее - 20-50 мкм и в отличие от частиц карбонильного железа имеют правильную сферическую форму; таковы, например, порошки Magnequench MQP-S-11-9-20001 [10].

Согласно литературе, каждая частица MQP-S-11-9-20001 - это плотный сферический «комок» из большого числа однодоменных нанозерен состава $\mathrm{Nd}_{2} \mathrm{Fe}_{14} \mathrm{~B}$. При этом каждое зерно в магнитном отношении очень похоже на частицу $\mathrm{CB}$, свойства которой описаны в разделах 1-3 выше. Поскольку МАЭ на основе $\mathrm{NdFeB}$ вызывают сейчас большой интерес, экспериментальных данных по ним накоплено много. Теоретические же исследования на начало 2017 года практически отсутствовали. Поэтому новизна, актуальность и приоритетность задуманной работы построение теории и моделирование кривых намагничивания таких систем были практически гарантированы.

\section{5. Магнитожесткая частица в МАЭ. Ориентационный гистерезис}

При изготовлении порошка Magnequench MQP-S-11-9-20001 внутри сферической капли металлического расплава инициируется многоцентровая кристаллизация, которая затем ингибируется, в том числе за счет быстрого охлаждения [10]. Таким образом получают частицы размером 20-50 мкм, состоящие из нанокристаллитов, каждый из которых - однодоменное магнитное зерно, то есть частица СВ. Поскольку формирование кристаллитов происходит при температуре, превышающей точку Кюри $\mathrm{NdFeB}$, и в отсутствие внешнего поля, то оси магнитной анизотропии зерен, составляющих частицу MQP-S-11-9-20001, распределены случайным образом. При обычном для теории предположении о том, что все зерна одинаковы, зависимость $M(H)$ такой частицы должна была бы (с точностью до масштаба) воспроизводить классическую кривую из работы [1] (см. рис. 7).
Однако фундаментальное отличие нашей постановки задачи от классики - и этим оправдывается эпиграф к статье - в том, что на этот раз сама частица (комок из большого числа случайно ориентированных зерен) не закреплена, но имеет возможность поворачиваться на произвольный угол. Тем самым, она получает расширенный набор возможностей реализовать состояние с наименьшей энергией: не только за счет переключения магнитных моментов внутри зерен, но и путем подстройки ориентации частицы как целого - ее поворота внутри упругой матрицы.

Положим - и это допущение можно подтвердить количественной оценкой, что элемент «одна частица в матрице» является вполне достоверной моделью структурной единицы изучаемого МАЭ, так что для описания свойств многочастичной системы достаточно просто суммировать вклады отдельных элементов.

Пусть на такой элемент накладывается сильное $\left(|\boldsymbol{H}|>H_{A}\right)$ поле, которое затем полностью выключается $(H=0)$. Поскольку исходной намагниченности у частицы нет (направления магнитных моментов зерен распределены случайным образом), то при первом намагничивании нет причины для ее поворота. Такая обработка, как объяснено в разделе 3 , сообщает частице магнитный момент $\boldsymbol{\mu}_{p}$, величина которого близка к половине предельно возможного (см. рис. 7), а ориентация примерно соответствуют направлению первого намагничивания. При включении поля в направлении, противоположном начальному (отрицательная область на рис. 7), оно создает момент сил $\left(\boldsymbol{\mu}_{p} \times \boldsymbol{H}\right)$, стремящийся повернуть вектор $\boldsymbol{\mu}_{p}$ частицы к текущему (в наших обозначениях - отрицательному) направлению $\boldsymbol{H}$. Если упругость матрицы мала, то частица развернется на угол, близкий к $180^{\circ}$, даже в относительно слабом поле: $|\boldsymbol{H}|<H_{A}$. Таким образом, перемагничивание будет обеспечено чисто механическим процессом при полном отсутствии внутренних переключений. Это означает, 
что в случае очень мягкой матрицы циклическое намагничивание МАЭ полностью обратимо во всем диапазоне, кривая $M(H)$ не содержит петель.

Для той же частицы в более жесткой матрице складывается иная ситуация. При наложении обратного поля частица начинает поворачивается по описанному выше сценарию, но затем упругость матрицы останавливает механический поворот. Для того чтобы продолжить перемагничивание требуется усилить поле. Тогда при наступлении условия $|\boldsymbol{H}| \geq \frac{1}{2} H_{A}$ вступят в действие внутренние степени свободы: магнитные моменты зерен, ориентированных под подходящими углами, начнут переключаться в направлении действующего поля. На этом этапе магнитный момент частицы (сумма моментов зерен) непрерывно изменяется по величине и направлению и, соответственно, изменяются условия баланса между моментом сил $\left(\boldsymbol{\mu}_{p} \times \boldsymbol{H}\right)$ и упругим моментом, создаваемым матрицей. В создавшихся условиях магнитное переключение зерен (внутренний процесс) и механический поворот частицы (внешний процесс) наиболее сильно влияют друг на друга и определить ход кривой намагничивания можно только с помощью численного расчета.

Увеличение поля до $H \sim H_{A}$ изменяет ситуацию. Для того чтобы понять, как при этом ведет себя частица, вспомним, что, с одной стороны, ее механический поворот вызывает накопление упругой энергии в матрице. С другой стороны, поле $H \geq H_{A}$ гарантирует перемагничивание любого из зерен, составляющих частицу, путем внутреннего переключения. Сколько-нибудь заметного поворота частицы не требуется, затрат упругой энергии практически нет. Применительно к частице, уже развернутой полем $H<H_{A}$ на значительный угол, рост поля до $H \geq H_{A}$ означает возможность вернуться в свое исходное положение, практически полностью разгрузив матрицу. При этом проекция магнитного момента частицы на направление поля заметно не изменит- ся, так как в поле $H \geq H_{A}$ ориентация осей анизотропии зерен не имеет значения. Расчет показывает, что такое возвратное движение происходит скачком.

Иллюстрацией этого эффекта (напомним, что $\xi=H / H_{A}$ ) служит рис. 8 , где показано угловое перемещение частицы, заключенной в достаточно мягкую матрицу; исходное положение принято за $0^{\circ}$. Начальное намагничивание частицы, где оси анизотропии зерен распределены случайно и равномерно, почти не меняет ее ориентацию (см. правый край графика). Первая половина цикла перемагничивания (она показана зелеными стрелками) начинается с уменьшения поля до нуля; при этом частица возвращается в начальное положение. В нарастающем по величине поле обратного направления (отрицательные значения $\xi$ ) частица поворачивается на значительный угол, а затем (когда внутри нее происходит массовое переключение зерен) резко разворачивается практически к начальному положению, «сбрасывая» механические напряжения в матрице. На второй половине цикла (когда $\xi$ варьируется от -2 до 2, см. сиреневые стрелки) ориентация частицы проходит все те же стадии, что и в первом полуцикле, с той лишь разницей, что стартовой точкой является состояние, где магнитные моменты всех зерен инвертированы. Поэтому на рис. 8 угловая «траектория» частицы во втором полуцикле намагничивания выглядит как зеркальное отражение той, что была пройдена за первый.

Из представленного анализа следует, что магнитный гистерезис в МАЭ должен сопровождаться также и внутрен-

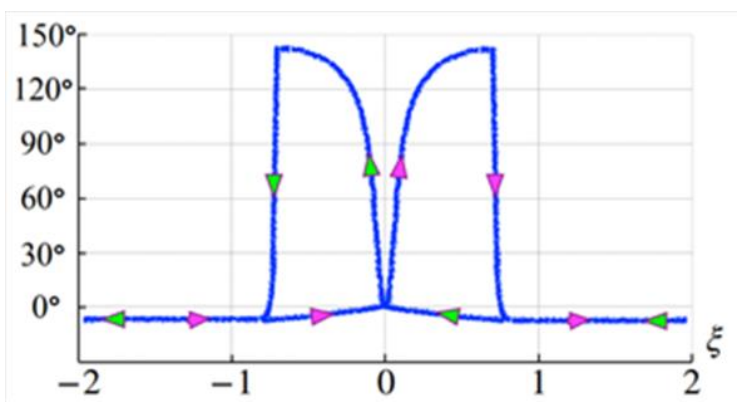

Рис. 8. Угол ориентации частицуы в мягкой матрицее при перемагничивании 
ним механическим. Однако магнитометрические эксперименты, хотя и способны обнаружить присутствие указанного эффекта, вряд ли могут быть адекватным методом его измерения. Для регистрации механического гистерезиса частиц, с учетом того, что он должен приводить, например, к разогреву МАЭ при многократном циклическом намагничивании, изменять спектр акустического сигнала от образца и т.п., скорее всего, нужно использовать другие способы.

Как можно видеть, например, из рис. 8 , в сильном поле $(\xi>1$, то есть $H \geq H_{A}$ ) связь между магнитным и механическим состояниями частицы теряется. В слабых же полях магнитное переключение зерен невозможно. Отсюда следует, что наиболее интересной для изучения областью является интервал $\left[\frac{1}{2} H_{A}<|\boldsymbol{H}|<H_{A}\right]$. Здесь в любой половине цикла - положительной или отрицательной - петля намагничивания формируется как результат совместного действия механических поворотов частицы целиком и «каскада» внутренних переключений составляющих ее зерен. Получаемые кривые (см. ниже) заметно отличаются от классической кривой для случайной системы частиц СВ в твердой матрице (см. рис. 7), и это отличие тем сильнее, чем мягче матрица.

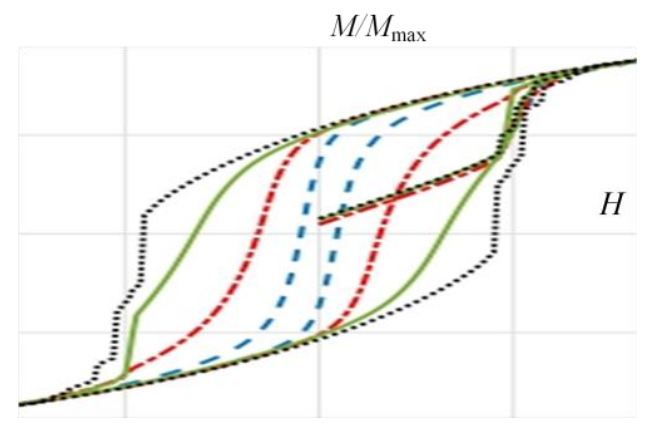

Рис. 9. Петли намагничивания МАЭ, полученные в работе [12] численным моделированием; снижение упругости матрицы приводит к сужению петель; самая широкая петля соответствует очень жесткой матрище, где повороть частии фактически исключены. Для двух петель показаньл линии первого (из состояния $M=0$ ) намагничивания

\section{6. Приложение модели к частицам в МАЭ}

Полный численный расчет величины $M$ - измеряемой в эксперименте проекции намагниченности образца МАЭ на направление приложенного поля - обсуждается в наших работах [11-13], технически он весьма сложен. Полученные с его помощью результаты иллюстрирует рис. 9, где показаны модельные кривые намагничивания, отвечающие различным значениям параметра $\kappa=E / K$, где $E$ - модуль Юнга матрицы; чем больше $\kappa$, тем шире петля. Для сравнения на рис. 10 в соизмеримом масштабе показаны петли, полученные при измерении циклического намагничивания в МАЭ, матрицей которого служил слабосшитый полидиметилсилоксан, а наполнителем - сферические частицы MQP-S-11-920001. Как видно, между петлями на рис. 9 и 10 есть полное качественное сходство, что говорит о фундаментальной адекватности предложенной модели. Прежде всего, она позволяет объяснить сужение петель при размягчении матрицы МАЭ. Это размягчение может быть или создано в одном и том же образце за счет нагревания, или, как в экспериментах [12], измерениями на серии образцов с одним и тем же содержанием частиц MQP-S-11-9-20001, но с различной степенью пластификации полидиметилсилоксановой матрицы.

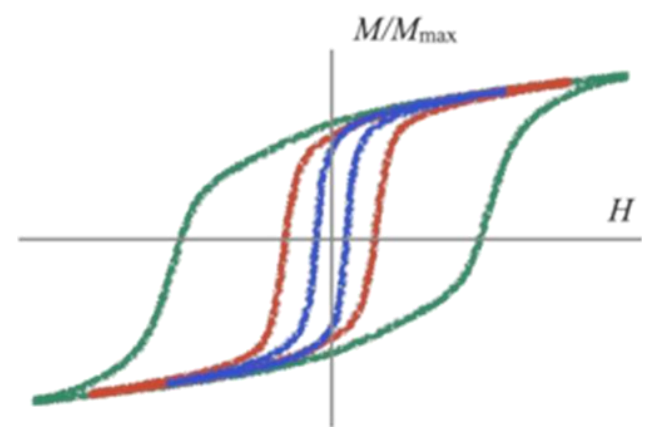

Рис. 10. Петли намагничивания МАЭ, наполненного сферическими частииами MQP-S-11-9-20001; снижение упругости матриџь приводит к сужению петель; кривые схематически воспроизводят результать измерений $[12,13]$. Самая широкая петля получена на твердой дисперсии, где матрицей служила эпоксидная смола 


\section{7. Заключительные замечания}

Главный результат представленной работы - это новое обобщение модели Стонера-Вольфарта. Построена теоретическая модель для описания магнитного отклика системы случайно ориентированных однодоменных зерен для случая, когда их относительные направления зафиксированы (частица - твердый комок), но вся система в целом механически подвижна. Именно решение этой необычной - и сильно усложненной по сравнению со стандартной - задачи (казалось бы, сугубая теория!) позволило впервые объяснить природу петель намагниченности реального магнитоактивного эластомера с магнитожестким наполнителем.

Построение базовой модели для нового типа МАЭ - это лишь один из первых шагов к достижению конечной цели: создать удобный алгоритм, который после задания набора параметров материала будет с хорошей точностью предсказывать поведение образца заданных разме- ров и формы в заданной комбинации магнитных полей и механических нагрузок. Этот длинный путь еще только предстоит пройти, и конечно, он не под силу одиночному коллективу. В последние годы исследования МАЭ самоорганизуются: на принципах взаимной дополнительности знаний и компетенций формируются цепочки научных групп, которые обеспечивают комплексный подход к проблеме: от вариантов синтеза через массированный сбор экспериментальных данных и их теоретического осмысления до конструирования прототипов устройств. Так, наша группа тесно сотрудничает с занятыми той же тематикой коллегами из России (Уральский федеральный и Московский государственный университеты, Государственный научно-исследовательский института химии и технологии элементоорганических соединений), Германии (Технические университеты Дрездена и Ильменау) и Австрии (Университет Вены).

\section{Библиографический список}

1. Stoner E.C., Wohlfarth E.P. A mechanism of magnetic hysteresis in heterogeneous alloys // Proceedings of the Royal Society (London) A: Mathematical, Physical and Engineering Sciences - 1948. - Vol. 240. - P. $599-642$.

2. Liu W., Liu Y., Skomski R., Sellmyer D. Nanostructured exchange-coupled magnets // Handbook of Advanced Magnetic Materials (Y. Liu, D. Sellmyer, D. Shindo, eds.) Advanced Magnetic Materials: Nanostructural Effects. Springer. - 2006. - Vol. 1. - P. 182-266.

3. Стоунер, Эдмунд Клифтон [Электронный ресурс]: Википедия. Свободная энциклопедия. URL: https://ru.wikipedia.org/wiki/Стоунер,_Эдмунд_Клифтон (дата обращения: 02.10.2020).

4. Stoner-Wohlfarth model [Электронный pecypc]: Википедия. Свободная энциклопедия. - URL: https://en.wikipedia.org/wiki/Stoner-Wohlfarth_model\#CITEREFStoner Wohlfarth1948 (дата обращения: 02.10.2020).

5. $М и$ Ч. Физика магнитной записи. - М.: Энергия, 1967.

6. Rigbi Z., Jilkén L. The response of an elastomer filled with ferrite to mechanical and magnetic influences // Journal of Magnetism and Magnetic Materials. - 1983. - Vol. 37. - P. 267-276.

7. Jolly M.R., Carlson J.D., Muñoz B.C., Bullions T.A. The magnetoviscoelastic effect of elastomer composites consisting of ferrous particles embedded in a polymer matrix // Journal of Intelligent Materials, Systems and Structures. - 1996. - Vol. 7. - P. 613-622.

8. Никитин Л.В., Миронова Л.С., Степанов Г.В., Самусь А.Н. Влияние магнитного поля на упругие и вязкие свойства магнитоэластиков // Высокомолекулярные соединения А. - 2001. - Т. 43. - С. 698-706.

9. Périgo E.A., Weidenfeller B., Kollár P., Füzer J. Past, present, and future of soft magnetic composites // Applied Physics Reviews. - 2018. - Vol. 5. - Art. No. 031301.

10. MQP-S-11-9-20001-070 [Электронный ресурc] - URL: mqitechnology.com/wp-content/uploads/2017/ 09/mqp-s-11-9-20001-070.pdf.

11. Vaganov M.V., Borin D.Yu., Odenbach S., Raikher Yu.L. Effect of local elasticity of the matrix on magnetization loops of hybrid magnetic elastomers // Journal of Magnetism and Magnetic Materials. 2018. - Vol. 459. - P. 92-97.

12. Vaganov M.V., Borin D.Yu., Odenbach S., Raikher Yu.L. Modeling magnetomechanical behavior of a multigrain magnetic particle in an elastic environment // Soft Matter. - 2019. - Vol. 15. - P. 4947-4960. 
13. Vaganov M.V., Borin D.Yu., Odenbach S., Raikher Yu.L. Mesomagnetomechanics of hybrid elastomer composites: Magnetization of elastically trapped particles // Journal of Magnetism and Magnetic Materials. 2020. - Vol. 499. - Art. no. 166249.

\title{
MAGNETICALLY ACTIVE ELASTOMERS: FERROMAGNETIC PARTICLES UNDER CONDITIONS OF RESTRICTED FREEDOM
}

\author{
Yu.L. Raikher, M.V. Vaganov \\ Institute of Continuous Media Mechanics UB RAS
}

\section{For citation:}

Raikher Yu.L., Vaganov M.V. Magnetically active elastomers: ferromagnetic particles under conditions of restricted freedom // Perm Federal Research Center Journal. - 2021. - № 1. - P. 6-18. https://doi.org/10.7242/2658-705X/2021.1.1

The article communicates certain results of research work carried out in 2017-2019. On the one hand, the authors didn't aim at going too deep into details; on the other hand, they didn't want to completely sacrifice physical rigour in favour of comprehensibility. For this reason, the original part is preceded by an introductory one (sections 1-3) that gives to an unprofessional reader a minimally sufficient amount of information on classical single-domain particle physics. It is also worth noting that the article for the first time presents in Russian the scientific materials that earlier were published only in international journals.

\section{Сведения об авторах}

Райхер Юрий Львович, доктор физико-математических наук, профессор, заведующий лабораторией физики и механики мягкого вещества, Институт механики сплошных сред УрО РАН - филиал Пермского федерального исследовательского центра УрО РАН («ИМСС УрО РАН»), 614013, г. Пермь, ул. Академика Королева, 1; e-mail: raikher@icmm.ru

Ваганов Михаил Викторович, младший научный сотрудник, лаборатория физики и механики мягкого вещества, «ИМСС УрО РАН»; e-mail: mikhail.vaganov.sci@gmail.com 